\title{
Research on simulated devices for Solar photovoltaic grid-connected generation system
}

\author{
Zhang quan-zhu, Ge yan-xiang, Deng yong-hong \\ College of electronic and information engineering, North China Institute of Science and Technology, 065201
}

\begin{abstract}
On the standpoint of energy conservation and emission reduction, one device simulated photovoltaic grid-connected generation system based on SPWM was designed in the paper. And DC/AC inverter could transduce efficiently direct current to alternating current. The MCU(Micro-Control-Unit), in this system could achieve the control method for maximum-power-point and tracking for frequency and phase. Moreover, the MCU could implement PWM (Plus-Width Modulating) through programming. The system showed clearly the whole photovoltaic grid-connected generation system using simulated methods and ways.
\end{abstract}

\section{Introduction}

With the rapid development of modern science and technology, the depletion of petrochemical energy and the impact on the environment are becoming increasingly serious, looking for renewable clean energy has become increasingly urgent global research topics. At present, the solar energy inexhaustible clean energy has been favored. The radiant energy of the sun can be converted into heat, which is a common use of solar energy. Photovoltaic power generation is the use of the energy directly into electrical energy the sun radiation, that is to say, light radiation can be converted into electrical energy by solar cells, after energy storage, control and transformation, which become direct or alternating current energy that can be used directly by people. The so-called grid connected, simply speaking, when voltage, phase, phase sequence and frequency are same, the principle of parallel operation can be achieved ${ }^{[1,2]}$.

\section{System structure and analysis}

As shown in Figure 1, Schematic diagram of photovoltaic grid connected analog device. The voltage $\mathrm{U}_{\mathrm{s}}$ generated by the solar panel, through the DC-AC power conversion, then the sine pulse width modulation wave (SPWM) is obtained, through the L-C consisting of sine wave filters, the filter to filter out high frequency harmonics, so that the sine wave fundamental voltage $\mathrm{u}_{01}$ is finally obtained, through the step-up grid connected transformer coupling, and provide voltage $u_{o}$ to the load. In the figure, $R_{s}$ is equivalent to the resistance of solar panels, $\mathrm{u}_{\mathrm{f}}$ is network synchronization voltage, that is a input of the control circuit coupled with the grid connected transformer, $\mathrm{u}_{\mathrm{REF}}$ is a given voltage for control circuit, and also is a instruction for the DC-AC converter when adjusting the voltage. 


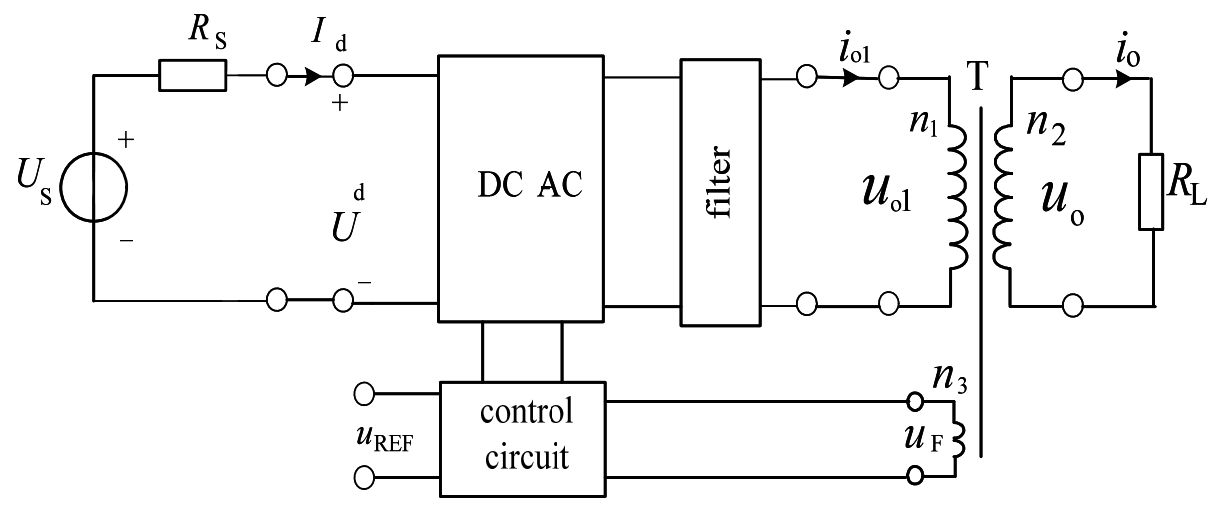

Fig. 1 Schematic diagram of photovoltaic grid connected analog device

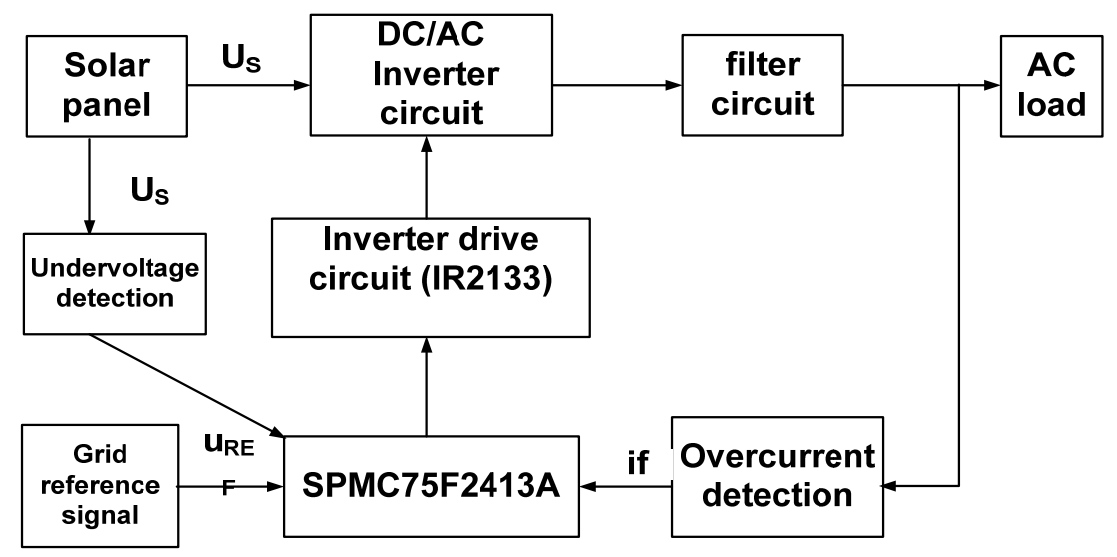

Fig. 2 the schematic diagram of the simulated devices for Solar photovoltaic grid-connected generation system

Figure 2 shows the schematic diagram of the simulated devices for Solar photovoltaic grid-connected generation system, The DC voltage Us which is output by the solar panel, through the DC/AC inverter circuit composed of IGBT, the SPWM wave is formed. L-C sinusoidal filter is used to filter the output waveform, and finally formed a relatively stable sine wave, then through the Boost-Type Grid-Connected transformer, the simulated devices be operated under load. Thereinto, the Sunplus 16bit microcontroller (SPMC75F2413A) as the core constitutes a control circuit, while completing the function of system protection, Photovoltaic Maximum Power Point Tracking. Through sampling the input voltage and output current of the system, the microcontroller forms the protection signal which is under voltage and over-current. At the same time, the maximum power point tracking is completed by sampling the input voltage; The reference voltage of the network is also input into the microcontroller through the detection circuit. As the reference voltage of the grid connected control, the frequency and phase tracking of the power grid is realized. The simulation scheme of the system is complete, the control and drive functions are complete, and the simulation operation can be carried out steadily and reliably.

\section{Inverter and filter main circuit}

Figure 3 shows the PV inverter simulation device and main filter circuit, inverter main circuit adopts the single-phase voltage type inverter circuit, two bridge arm consisting of four IGBT IGBT, Infineon produced by IKA15N60T, the IGBT with anti parallel diode, the main circuit connection more simple. In addition, the maximum emitter voltage of the IGBT is $600 \mathrm{~V}$, the maximum collector current is $15 \mathrm{~A}$, and the maximum junction temperature is $175 \mathrm{DEG} \mathrm{C}$, which meets the application requirements. Through the drive circuit of the 4 wave SPWM wave drive to its grid, to achieve the DC/AC 
transform, output SPWM wave, through the SPWM wave voltage and frequency adjustment ${ }^{[3,4]}$. duty cycle $\mathrm{D}$ and frequency f control to realize the output

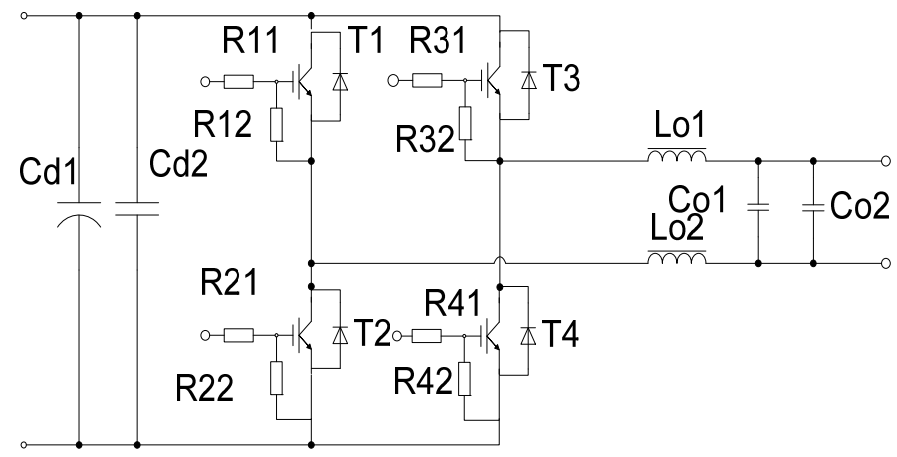

Fig. 3 inverter and filter main circuit of photovoltaic grid connected analog device

\section{3 drive circuit}

In this design, we use IR (International, Rectifier) company's IR2133 chip as the driving circuit to drive IGBT (IKA15N60T). IR2133 is designed for high voltage, high speed power switching devices MOSFET and IGBT, which has the advantages of simple and convenient, especially suitable for three-phase Variable frequency transformer circuit.

\subsection{IR2133 drive circuit}

IR2133 is a driver chip, when it needs PWM pulse input, it will produce pulse output, IR2133 input pulse is provided by 16bit microcontroller SPMC75F2413A. The input signal of the IR2133 is compatible with the output signal of the CMOS or TTL circuit of $5 \mathrm{~V}$, and the maximum voltage of the output signal can reach $20 \mathrm{~V}$, so that the driving ability of the driving signal is obviously enhanced, and the purpose of driving the IGBT reliably is achieved. In addition, the input control circuit also provides the dead time for high-end devices and low-end devices with one arm in the chip, in order to avoid the phenomenon of simultaneous misoperation that the two powers element is driven with one arm during the switching transition, Thus, the reliability of the drive pulse is improved. drive circuit based on IR2133, which outputs 6 pulse signals, is used to drive the IGBT three-phase inverter.IR2133 based IGBT inverter drive circuit. The output of the systemic inverter circuit is a single phase AC voltage, only 4 drive signals are enough, and the redundant 2 signals can be suspended or grounded through the $10 \mathrm{k} \Omega$ resistor. If the protection function is not needed during the application of IR2133, each protective terminal can not be processed, which is equivalent to that the IR2133 has been kept in the normal working state.

\section{4 system detection circuit}

The design of the detection circuit is very important, and it directly affects the accuracy of the data acquisition by the microcontroller. The system has five detection circuits, which respectively realize the functions of frequency tracking, maximum power point tracking, under voltage protection, overcurrent protection and phase sampling . The frequency tracking detecting circuit transforms the sine wave into the rectangular wave of the same frequency, input the rectangular wave to the microcontroller, and the time difference is obtained by the zero crossing of the detecting waveform, Thus, the frequency of the voltage signal is obtained, and frequency tracking is realized. The sampling circuit of maximum power point tracking is based on the features of maximum power output, When the load equivalent resistance is equal to the load internal resistance, the power output power is the maximum. use the matching method of the sampling voltage values, If the supply voltage $60 \mathrm{~V}$ is constant (output voltage of solar panels),after the load is connected, the full bridge type inverter voltage regulation is used, in order to make the load equivalent resistance equal to the internal resistance of the power supply, then the Load obtain the output voltage $U_{d}$, should be equal to half of the power supply voltage, to realize the function of maximum power point tracking (MPPT). 


\section{1 frequency detection circuit}

As shown in Figure 4 for the frequency detection circuit, the detected AC input voltage, through the differential amplifier circuit the which consists of integrated operational amplifier (TL082), according to the resistance in the figure, calculated the difference signal magnification is 50 , the output voltage of the operational amplifier is $50\left(\mathrm{u}_{1}-\mathrm{u}_{2}\right)$. As the supply voltage of TL082 is: $\pm 15 \mathrm{~V}$, the maximum output voltage of the amplifier is: $\pm 15 \mathrm{~V}$. When the input peak-to-peak voltage is twice the grid reference voltage $U_{\text {ref }}$, the output voltage signal is turned off and the actual output voltage is a square wave. After the square wave passes through the diode IN4148,

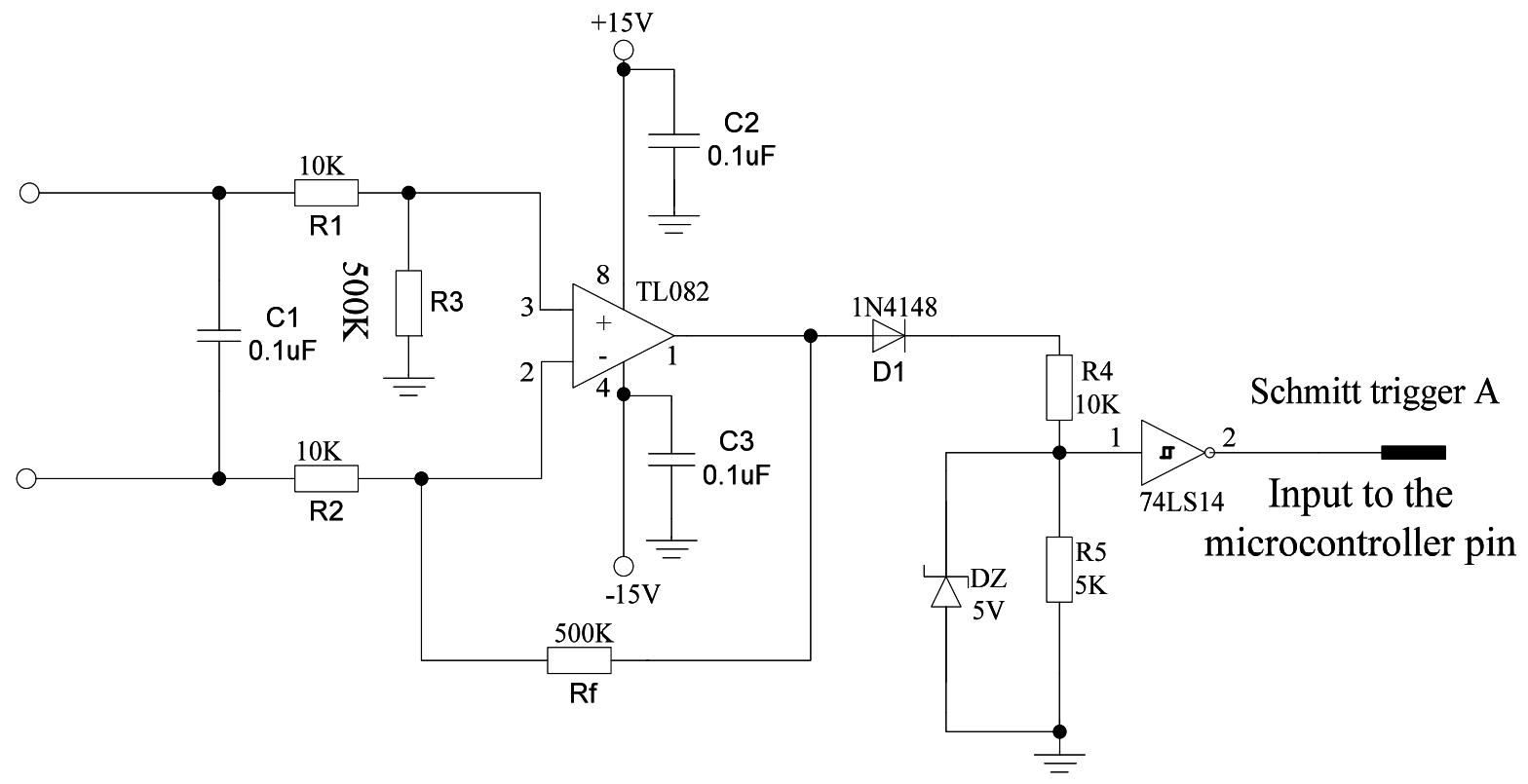

Fig.4 frequency detection circuit

\section{2 voltage and current detection circuit.}

The detection of voltage and current is almost similar. The voltage detection circuit is shown in figure 5. volts, where the use of the proportional operation is the buck operation, the voltage signal is reduced to less than $5 \mathrm{~V}$, so that the microcontroller can accurately sample. The maximum power point tracking (MPPT) sampling circuit is basically the same as the voltage detection circuit, which has been described previously.

The current detection circuit is that a small resistor is connected in the current detecting circuit, such as milli ohm resistance, using the same differential amplifying circuit, the voltage signals at both ends of the ohm resistor are detected, adjusted and input to the the negative half-cycle of the original waveform is eliminated, only the positive half-cycle rectangular wave is output. which laid the foundation for the Sunplus single chip microcomputer accurately collecting the zero crossing of waveform. Thus, the frequency of the original sine wave is detected. Sunplus MCU regulates the output AC frequency of the inverter circuit by adjusting the output SPWM wave. which realize that the frequency of the output voltage is equal to the frequency of the reference signal of the power grid, and the voltage frequency of the power grid can be automatic tracked . microcontroller, after calculation of microcomputer, corresponding to calculate the current value.

As shown in Figure 6, the current detection circuit. The operational amplifier uses the independent operational amplifier of TL082, which can avoid mutual interference between the sampling circuits, so that the output signal is accurate and anti-interference is strong. It is worth noting that the sampling circuit must be repeatedly debugged, to make the amplitude, stability and smoothness of the waveform determine, only in this way can the detection signal be input to the microcontroller to ensure the accuracy of the signal. 


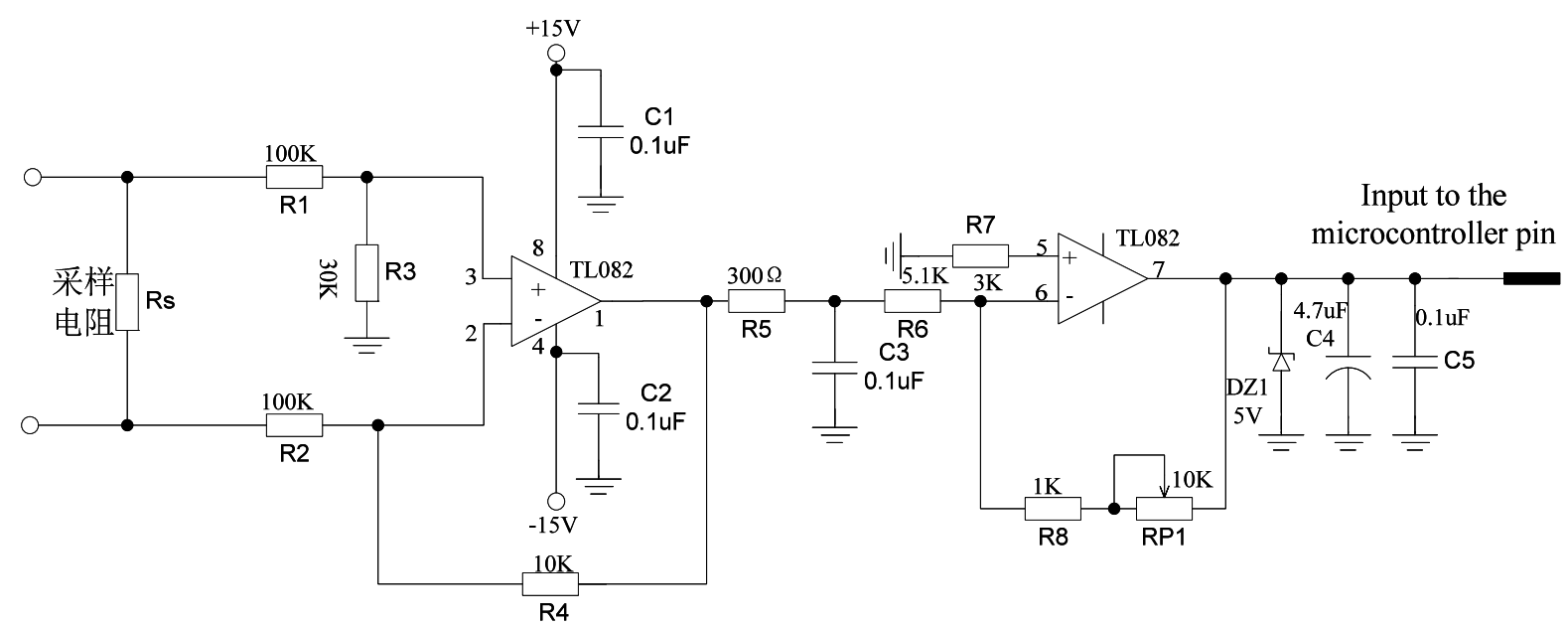

Fig.5 voltage detection circuit

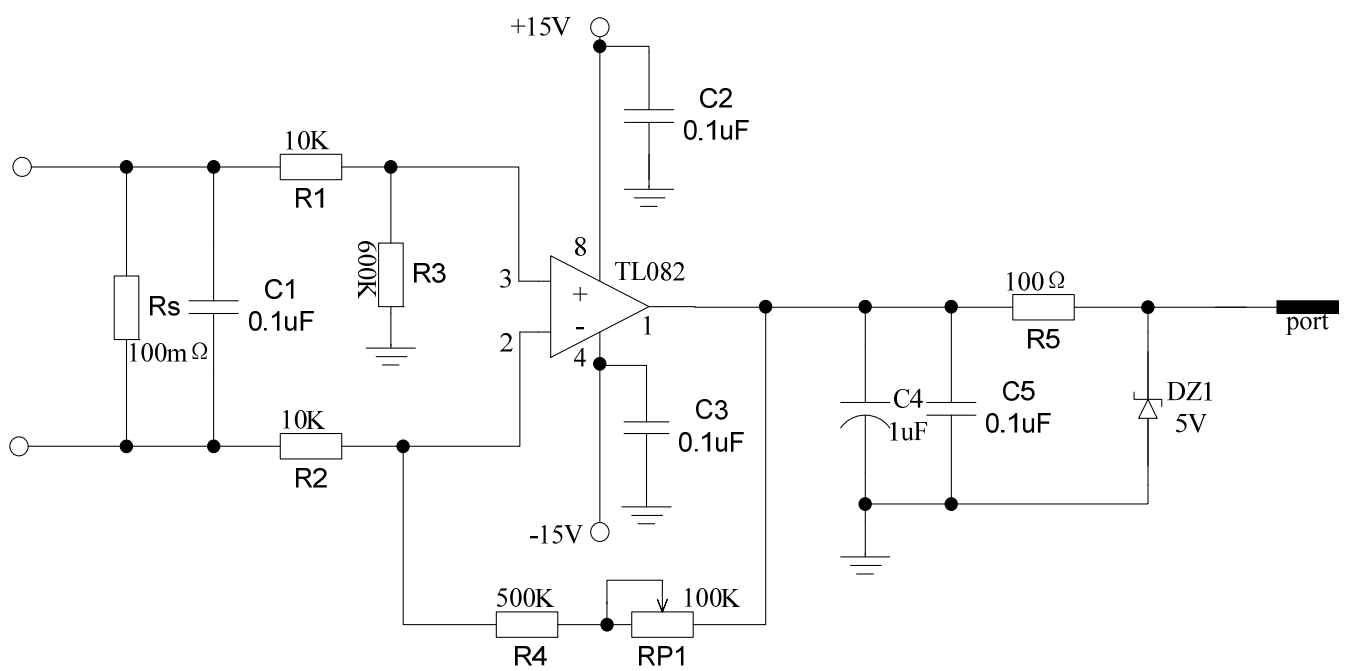

Fig.6 current detection circuit

\section{Conclusion}

The device is a simulation device, which uses the voltage source to simulate the photovoltaic solar cell, so some places need to be perfected. For example, the actual maximum power point tracking (MPPT) is more complex, need to consider many factors of light intensity, temperature,

\section{references:}

[1] Dong Mi, Luo an. Design and control method of inverters in grid connected photovoltaic systems [J]. automation of electric power systems, 2006 (20): 10-13;

[2] Zhang Zhi-qiang, Ma Qin, Cheng Da-zhang. research on the control technology of solar photovoltaic power generation system $[\mathrm{J}]$. Low voltage humidity. but in the simulation device, Just focusing on simulating the intensity of the light (such as when the maximum output power is reached, the load resistance value is equal to the resistance value in the power supply), and other factors is ignored. We are still actively studying and have achieved more new results.

apparatus, 2008. (12):28-31;

[3] Zhao Zhen-ming. Solar photovoltaic power generation and application $[\mathrm{M}]$, Science Press, 2005,10;

[4] Liu Dao-chun, solar photovoltaic technology and its development prospects [J], public power, 2009 (03): 16-18. 
[5], Hou Guo-qing, Chen Shi-hua. $20 \mathrm{~kW}$ grid connected photovoltaic power generation system $[\mathrm{J}]$, solar energy, 2008 (11): 44 - 46.

Foundation item: the project by science and Technology Department of Hebei province
(1627460316214408); Chinese Coal Industry Association (MTKJ2016-35, MTKJ2016-329); fundamental research funds for the central universities such as funding (3142016022, 3142017044) 